\title{
Production and dissipation of kinetic energy in grid turbulence
}

\author{
Wouter J. T. Bos 투 \\ Univ Lyon, École Centrale de Lyon, INSA Lyon, Université Claude Bernard Lyon 1, \\ CNRS, Laboratoire de Mécanique des Fluides et d'Acoustique, UMR 5509, \\ 36 Avenue Guy de Collongue, F-69134 ECULLY, France
}

(Received 11 April 2020; accepted 23 September 2020; published 19 October 2020)

\begin{abstract}
In grid turbulence, not so far behind the grid, an average flow can be observed with a close to sinusoidal velocity profile, corresponding to the wakes behind the grid bars. The kinetic energy of this mean flow decays rapidly and a close to isotropic flow is observed further downstream. We show how these wakes behind the grid bars influence the downstream turbulence. In particular, we investigate the decay rate of kinetic energy, the behavior of the normalized dissipation rate, and the sensitivity of the flow on initial conditions. We show that the initial value of the ratio of the length scale of the turbulence to the mesh-size determines the precise decay of the mean-flow and the generation of the turbulent kinetic energy. We further show how a simple turbulence model can estimate the degree of nonequilibrium and inhomogeneity of grid turbulence and how this model can be extended to take into account disequilibrium observed in direct numerical simulations of decaying isotropic turbulence.
\end{abstract}

DOI: 10.1103/PhysRevFluids.5.104607

\section{INTRODUCTION}

Since the first experiments of Simmons and Salter [1] (see also Ref. [2]), the turbulent motion behind a grid in a wind-tunnel has been investigated intensively in a large number of investigations to study a variety of applications, such as wind energy [3,4], scalar mixing [5-7], transport of particles [8,9], superfluid flows [10], or to study intrinsic features of the turbulence itself [11-15]. This summary mentions only a fraction of the immense literature on grid-turbulence.

The main reason for its popularity is that this type of flow is experimentally close to the academic reference case of isotropic turbulence. Historically, grid-turbulence experiments were therefore carried out avoiding systematically the region close to the grid, where a nonisotropic and inhomogeneous production of kinetic energy by shear-layers behind the grid bars is present. For instance, in early work [16] it was mentioned: "The range $x / M<20$ is of course excluded from the system of classification since the turbulence takes a little time to become uniform (in the lateral direction) and isotropic." $M$ is here the mesh-size, and $x$ is the distance from the grid in the streamwise direction. Consequently, most studies on grid-turbulence investigated this far downstream evolution (see, e.g., Refs. [11,12,17]).

However, in recent work [18] it was recognized that this zone, closer to the grid might be of interest, since it allows the investigation of a transition region between a flow where production and dissipation are both important, and a freely decaying, productionless flow further downstream. In this region several interesting observations were made which were not in complete agreement with classical pictures of self-similar decaying turbulence (e.g., Ref. [19]). Since then, critical assessment by simulations and experiments of decaying grid-turbulence closer to the grid has opened the debate of the nature of this nonequilibrium turbulence and its dependence on the grid-type [20-23].

One of the observations in the recent grid turbulence experiments [18] is that the normalized dissipation rate is not constant in the near grid region. The normalized dissipation rate is 
defined by

$$
C_{\epsilon}=\frac{\epsilon \mathcal{L}}{\mathcal{U}^{3}},
$$

with $\mathcal{L}$ the integral length scale, $\epsilon$ the dissipation of kinetic energy and $\mathcal{U}$ the RMS velocity fluctuation. In a recent work we explained some of the observations using a perturbation analysis of the energy flux around an equilibrium state [24]. However, a full picture, including the influence of the shear layers on the turbulence production and their influence on the decay rate of the turbulent kinetic energy further downstream has not been given yet, except from a tentative study using the EDQNM turbulence model [25]. The goal of the present investigation is to understand this transition zone, close to the grid for the case of high-Reynolds number turbulence. We will show how the kinetic energy of the turbulence is produced by the shear layers, and how the kinetic energy and its statistical inhomogeneity evolves as a function of time.

This investigation will show that several salient features of near grid-turbulence, as observed in recent investigations can be reproduced by one of the simplest existing turbulence models. In particular, we shall answer to several of the standing questions, as summarized in the list of future issues, which was dressed in Ref. [18]:

(1) What causes the sudden transition from the power-law evolution of $C_{\epsilon}$ to an (almost) constant value?

(2) What is the downstream distance where this happens?

(3) How can this downstream distance be estimated in terms of the geometry of the turbulence generator and inlet Reynolds number?

In addition, we will answer the following questions:

(1) How does the peak of the kinetic energy relate to the total kinetic energy injected in the flow?

(2) How does the peak of the kinetic energy and its downstream distance from the grid depend on the flow properties?

We can summarize our main objectives as follows: how does grid-turbulence depend on initial conditions and how can we model the behavior?

In Sec. II we will show how the mean-field behind the grid bars can be described in a simple manner. This mean-field loses its energy to the turbulent fluctuations, and this interaction is described and modeled in Sec. III. In Sec. IV we show how nonequilibrium can be assessed within a two-equation turbulence model. The cross-stream inhomogeneity of the kinetic energy is addressed in Sec. V. The model which describes the interaction of the averaged grid-wakes and the turbulent fluctuations is numerically integrated in Sec. VI for experimentally relevant parameters. In Sec. VII it is shown how at short-times the amount of generated kinetic energy can be understood by comparing the dynamics with homogeneous shearflow. The extension of the model to be able to describe disequilibrium in direct numerical simulations of decaying isotropic turbulence is presented in Sec. VIII. The results allow us to answer the above-stated open questions, which are replied upon in the conclusions, Sec. IX.

\section{A SIMPLIFIED DESCRIPTION OF THE MEAN FLOW}

In the present work we will decompose grid-generated turbulence into a mean part (the wakes behind the grid bars) and a fluctuating part [26], and we will investigate how the interaction between the energy in these two components of the flow is influenced by initial conditions.

The mean flow indicated by $\bar{U}_{i}$ is assumed unidirectional [in the streamwise $(x)$ direction] and varies in the cross-stream (y) direction. Obviously there are two cross-stream directions in the flow behind a regular square-grid, but given the further approximations we introduce, considering one direction is a fine enough description. We will measure the velocity-field in the frame of reference moving with the wind-tunnel average speed $U_{0}$, so that the mean-flow $\bar{U}_{i}$ is zero when averaged over the cross-stream direction. A sketch of the flow is given in Fig. 1. 


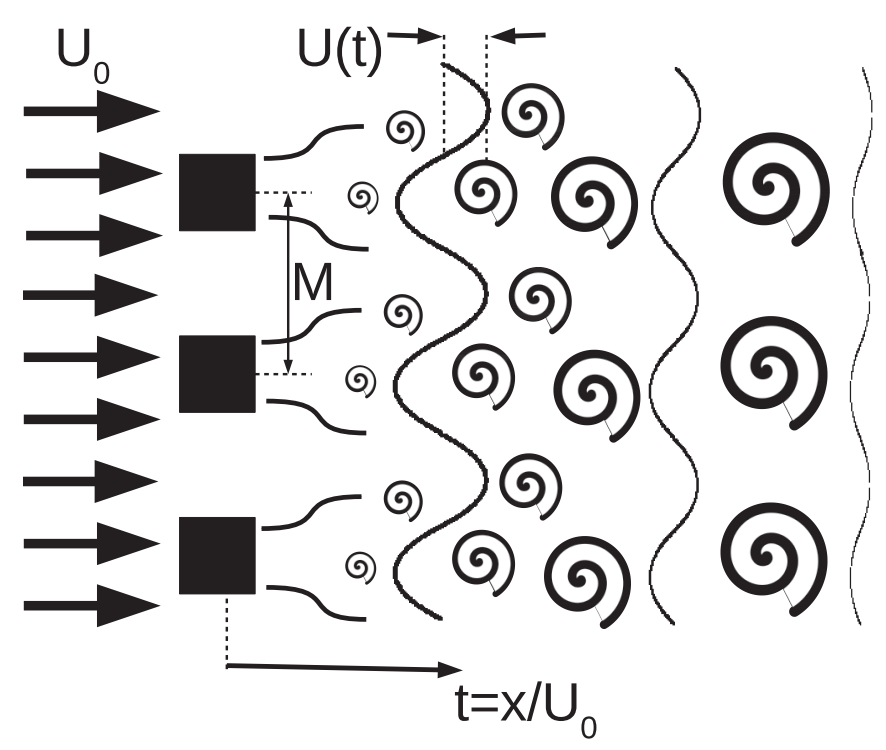

FIG. 1. Sketch of the geometry considered in the present investigation. A uniform incoming flow $U_{0} \boldsymbol{e}_{x}$ is perturbed by a grid. The resulting flow is decomposed into a sinusoidal mean profile $U(y)=\overline{U_{x}}-U_{0}$ and a fluctuating part.

The mean-flow is thus given by

$$
\overline{\boldsymbol{U}}(\boldsymbol{x}, t)=\bar{U}(y, t) \boldsymbol{e}_{x}
$$

We now consider the case of regularly spaced grid bars as used in the majority of studies on gridturbulence. The spacing of the bars is $M$ (the mesh length). The mean flow behind the bars will then be periodic with a spatial period $M$. To some approximation we can then model the mean flow by

$$
\bar{U}(y, t)=U(t) \sin \left(\frac{2 \pi y}{M}\right)
$$

This can be shown to be a good approximation even for more complicated passive turbulencegenerating grids [27]. Indeed, behind fractal grids the wakes were also shown to rapidly converge to a sinelike shape.

Clearly Fig. 1 does not represent all possible shapes of grids. However, what we argue is that all passive grids, fractal or not, will through their blockage generate shear-layers. Most grids will have some spatial, large-scale periodicity, which can be grossly modeled by a sine-function. The case of active grids $[28,29]$ is particular since in this case, in addition to blockage, the rotating shafts of the grid will induce a large transverse turbulent diffusion. The sinelike profiles are probably less prominent for such grids if the stirring is intensive. We will come back to this issue later.

By prescribing the shape of the mean-flow, we have simplified the problem considerably. It will now be possible to describe the interaction of the mean flow with the fluctuating turbulent field in a fairly simple manner. We consider the kinetic energy of the mean field, averaged over one mesh length,

$$
K=\frac{1}{M} \int_{0}^{M} \frac{1}{2} \bar{U}(y, t)^{2} d y=\frac{1}{4} U(t)^{2}
$$


The evolution of this quantity is simply derived from the Reynolds averaged Navier-Stokes equations (see Appendix A),

$$
\dot{K}=-p-D
$$

where

$$
p=-\frac{1}{M} \int_{0}^{M} \overline{u v} \partial_{y} \bar{U} d y,
$$

and

$$
D=\frac{1}{M} \int_{0}^{M} v\left(\partial_{y} \bar{U}\right)^{2} d y,
$$

with $\overline{u v}$ the Reynolds stress. The contribution of the turbulent diffusion in the cross-stream direction vanishes in the energy balance since its integral over a period $M$ is zero (see Appendix A, where we derive this average balance from the pointwise evolution equations).

The fluctuating part of the turbulence, i.e., the turbulent flow that is studied in most investigations of grid-turbulence, is governed by the balance (see Appendix A)

$$
\dot{k}=p-\epsilon,
$$

where

$$
k=\frac{1}{M} \int_{0}^{M} \frac{1}{2} \overline{u_{i} u_{i}} d y
$$

and

$$
\epsilon=\frac{1}{M} \int_{0}^{M} v\left(\overline{\partial_{j} u_{i} \partial_{j} u_{i}}+\overline{\partial_{j} u_{i} \partial_{i} u_{j}}\right) d y,
$$

where $k$ and $\epsilon$ are the kinetic energy and dissipation rate of kinetic energy, both averaged over a grid-period. The second part of the dissipation in Eq. (10) is the inhomogeneous part, which vanishes in this description, where the turbulence is assumed periodic in the $y$ direction and homogeneous in the $x$ and $z$ directions in the moving reference frame.

We have therefore at this point a flow-field, described by the two quantities $k$ and $K$, and their equations contain the unknown terms $\overline{u v}$ and $\epsilon$. We note that the formulation is in particular simple because we consider the kinetic energy budgets averaged over a grid-period, and not pointwise, so that the turbulent diffusion terms vanish. This simplification is no approximation, but a consequence of the periodicity of the flow. To proceed we need to introduce a model.

\section{MODELING THE TURBULENCE PRODUCTION}

The most widely known turbulence model is the $k-\epsilon$ model [30]. To describe the turbulence, equations are written for the quantities $k$ and $\epsilon$. In addition to its simplicity, it is known to give good results both in self-similar decaying turbulence and in shear-flows, even though some precise quantitative features are mispredicted [31]. Since we consider the transition zone between a simple shear-flow and a freely decaying flow, the $k-\epsilon$ model seems to be the ideal starting point for our investigation. Also, even though the $\epsilon$ equation is often critized as being nonphysical, in the case of homogeneous turbulence in the presence of shear it can be derived using some plausible assumptions [32].

We stress here that the goal is not to model the detailed flow around a grid bar, where vortexshedding and different types of flow structures depend on the detailed nature of the bar shapes. In this very-near region, the exact modeling of the flow by simple engineering turbulence models is very challenging. To correctly describe this region one needs a detailed description, taking into account the influence of instationarity and anisotropy. In the present approach we focus on the 
periodic mean flow which is observed somewhat behind it. We admit therefore the existence of the mean-flow, without attempting to accurately capture its genesis.

The $k-\epsilon$ model is an eddy-viscosity closure, where the Reynolds stresses are modeled by

$$
\overline{u v}=-v_{T} \partial_{y} \bar{U} .
$$

The eddy viscosity $\nu_{T}$ is modeled as a function of $k$ and $\epsilon$,

$$
v_{T}=C_{\nu} \frac{k^{2}}{\epsilon}
$$

The kinetic energy of the mean flow (constituted by the shearlayers behind the grid) is determined by relation Eq. (5). The $k-\epsilon$ equations reduce in our description to Eq. (8) for the turbulent kinetic energy and the $\epsilon$ equation,

$$
\dot{\epsilon}=\frac{\epsilon}{k}\left(C_{\epsilon 1} p-C_{\epsilon 2} \epsilon\right)
$$

with

$$
p=C_{v} \frac{k^{2}}{\epsilon} \frac{1}{M} \int_{0}^{M}\left(\partial_{y} \bar{U}\right)^{2} d y .
$$

In principle, not only the mean flow is $y$ dependent, but also $k$ and $\epsilon$ are. The above expression implicitly assumes a homogeneous distribution of $k$ and $\epsilon$. The error induced by this assumption is estimated in Appendix B and does not exceed a few percents in the present case. The inhomogeneous profile of the kinetic energy in the $y$ direction is further considered in Sec. V.

To close the system, we need to determine the average squared shear which appears in Eqs. (7) and (14) for $D$ and $p$, respectively. We can obtain this quantity exactly by averaging the squared gradient of the mean velocity profile given by Eq. (3) over the $y$ direction,

$$
\frac{1}{M} \int_{0}^{M}\left(\partial_{y} \bar{U}\right)^{2} d y=\frac{2 \pi^{2}}{M^{2}} U^{2}=\frac{8 \pi^{2}}{M^{2}} K .
$$

Thereby, we have reduced our problem to the three ODEs,

$$
\begin{aligned}
& \dot{K}=-\frac{k^{2}}{\epsilon} \frac{K}{\tilde{M}^{2}}\left(1+\frac{C_{\nu}^{-1}}{\operatorname{Re}}\right), \\
& \dot{k}=\frac{k^{2}}{\epsilon} \frac{K}{\tilde{M}^{2}}-\epsilon, \\
& \dot{\epsilon}=\frac{\epsilon}{k}\left(C_{\epsilon 1} \frac{k^{2}}{\epsilon} \frac{K}{\tilde{M}^{2}}-C_{\epsilon 2} \epsilon\right),
\end{aligned}
$$

where $\tilde{M}=M /\left(\sqrt{8 C_{\nu}} \pi\right)$ and $\operatorname{Re}=k^{2} /(\epsilon \nu)$. In the following we will consider the high Reynolds number case, where the last term in brackets of Eq. (16) is negligible. Evidently, since $k$ and $\epsilon$ are positive quantities, all quantities will eventually decay. We have therefore an initial value problem depending on the values of $K, M, k$, and $\epsilon$. Since we consider the high Reynolds number case, the viscosity does not enter the system.

Further insights into this system are obtained by rewriting the equations of $k$ and $\epsilon$ in their original form, and the equation of the mean field as an equation for $p$

$$
\begin{aligned}
& \frac{\dot{k}}{k}=\frac{\epsilon}{k}\left(\frac{p}{\epsilon}-1\right), \\
& \frac{\dot{\epsilon}}{\epsilon}=\frac{\epsilon}{k}\left(C_{\epsilon 1} \frac{p}{\epsilon}-C_{\epsilon 2}\right),
\end{aligned}
$$




$$
\frac{\dot{p}}{p}=\frac{\epsilon}{k}\left(\left(2-C_{\epsilon 1}\right) \frac{p}{\epsilon}-\left(2-C_{\epsilon 2}\right)-\frac{k^{3}}{\epsilon^{2} \tilde{M}^{2}}\right) .
$$

A salient feature of this representation is that, apart from $k, p, \epsilon$, the dynamics of the production depend explicitly on the square of a length-scale ratio,

$$
\alpha_{L}(t) \equiv \frac{k(t)^{3 / 2}}{\epsilon(t)} \frac{1}{\tilde{M}} \sim \frac{L(t)}{M},
$$

where we introduce a length scale $L \sim k^{(3 / 2)} / \epsilon$, characterizing the turbulence. For given values of $k, p, \epsilon$, the dynamics can thus be different depending on the initial value of $\alpha_{L}$. Clearly, this term in the equations shows how the initial conditions explicitly affect the decay of grid turbulence. We note here that in the experiments [18], it is also a ratio of a local parameter and a parameter linked to the initial conditions which pilots the behavior of the turbulence. A similar observation was made in Ref. [25], where a timescale ratio was identified which quantifies the influence of the initial conditions. We note in this context that $\alpha_{L}^{2}$ can also be interpreted as a timescale ratio, and we will come back to this in Sec. VII.

It has long been argued that initial conditions might influence a freely evolving turbulent flow for a long time [33]. The present description shows how this influence shows up in the evolution equations of the turbulent kinetic energy. In real experiments a drastic way to increase the initial value of this parameter is to use active grids [28,29]. Indeed, in active grids the typical length scale of the turbulence is largely increased, since instead of developing gradually from instabilities in the grid-wakes, the turbulent kinetic energy is immediately generated by the grid, at the expense of the energy of the mean wake-flow. In the present investigation, to illustrate the importance of the initial conditions on the turbulence, we will assess the influence of $\alpha_{L}$ on the downstream turbulence.

\section{NONEQUILIBRIUM SCALING OF UNSTEADY TURBULENCE}

In the absence of production, the $k-\epsilon$ model depends only on two parameters, $k$ and $\epsilon$. Therefore, the turbulence is represented by a single length scale, determined by relation Eq. (1), or equivalently, a single timescale $k / \epsilon$. In unsteady flows, another timescale can be defined, determined by the time-variation of the dissipation rate,

$$
\tau_{\epsilon}=\left(\frac{\dot{\epsilon}}{\epsilon}\right)^{-1} .
$$

It is this second timescale that becomes important when there is a significant imbalance between production and dissipation.

In our recent theory [24] we acknowledged the importance of this second timescale in the description of unsteady turbulence. We decomposed a turbulent flow into an equilibrium part, consisting in the turbulence which would result when an equilibrium is established between production and dissipation $(p-\epsilon=0)$, and the remaining, disequilibrium part $\tilde{k}$. In the equilibrium part of the flow, the normalized dissipation [Eq. (1)] yields a constant value $C_{\epsilon 0}$.

We found for the ideal case of high Reynolds number isotropic turbulence a relation between the equilibrium and nonequilibrium parts, given by

$$
\frac{\tilde{k}}{k}=\frac{2}{9} \frac{\dot{\epsilon} k}{\epsilon^{2}}
$$

[This relation is straightforwardly obtained from the theoretical results [24] using their Eq. (13), Eq. (21), and the integral from $\kappa_{0}$ to $\infty$ of Eq. (10).] We use this relation here in the context of turbulence behind a grid, which is not strictly isotropic. The isotropic contribution to the energy cascade is however expected to be dominant with respect to anisotropic contributions in grid-turbulence and the above expression should at least give a good order of magnitude estimate of 
the imbalance. Furthermore, when the shear-layers have decayed sufficiently, the resulting flow is near isotropic (far enough behind the grid), so that the isotropic approach is at least justified there.

Since relation Eq. (34) depends on $\dot{\epsilon}$, for which the $k-\epsilon$ model gives an expression [Eq. (13)], we find that

$$
\frac{\tilde{k}}{k}=\frac{2}{9}\left(C_{\epsilon 1} \frac{p}{\epsilon}-C_{\epsilon 2}\right) .
$$

Furthermore, we showed that the normalized dissipation can be expressed as a function of this perturbation, leading to the relation

$$
\frac{C_{\epsilon}}{C_{\epsilon 0}}=\left(1+\frac{\tilde{k}}{k}\right)^{-\frac{15}{14}} .
$$

In the following we will use the $k-\epsilon$ model to compute $k$ and $\epsilon$, and we will determine the unsteady perturbation $\tilde{k}$ a posteriori, using Eq. (25). This will allow us to estimate the nonequilibrium within the context of a two-equation turbulence model. We note here that this is possible in a two-equation model, since we have access to the values of $k, \epsilon$, and $\dot{\epsilon}$. An even more reduced description, using only one equation [34] does not provide enough information on the turbulence to assess the imbalance in this way.

Before proceeding to a numerical assessment of the model we can already estimate the nonequilibrium in the limiting case $p=0$. For this case, Eq. (25) yields $|\tilde{k} / k|=0.43$. The perturbation $\tilde{k}$ does therefore not become excessively large and never exceeds $43 \%$ in the region beyond the peak of the turbulent kinetic energy. Treating the nonequilibrium as a perturbation is therefore not unjustified on these grounds.

\section{CROSS-STREAM INHOMOGENEITY}

It is possible within the present framework to estimate the inhomogeneity of the turbulence. For this we write the kinetic energy balance without cross-stream averaging. Let us for simplicity indicate the ensemble averaged kinetic energy, which has not been averaged over the cross-stream direction, by $k(y)=\overline{u_{i} u_{i}} / 2$, so that

$$
k=M^{-1} \int_{0}^{M} k(y) d y .
$$

The evolution equation for $k(y)$ writes

$$
\partial_{t} k(y)=p(y)+d(y)-\epsilon(y) .
$$

In this section the explicit dependence on $y$ shows that ensemble averaged quantities are considered which are not averaged over the cross-stream direction. The exact expressions of the different contributions can be found in Appendix A. The term $d(y)$ contains nonlinear (turbulent) diffusion, pressure diffusion, and viscous diffusion. The term $\epsilon(y)$ is the dissipation. Within the $k-\epsilon$ model $d(k)$ is modeled as

$$
d(y)=\partial_{y}\left\{\left[v_{T}(y)+v\right] \partial_{y} k(y)\right\}
$$

so that its cross-stream integral yields zero contribution. The viscous contribution will be neglected, in line with the experimental observation [27] where the dominant terms determining the crossstream inhomogeneity are the production and turbulent transfer. The production $p(k)$ is given by Eq. (14) without the streamwise averaging, yielding, using Eq. (3),

$$
p(k)=\frac{8 \pi^{2} K}{M^{2}} v_{T}(y)[1+\cos (4 \pi y / M)] .
$$

We see that the frequency of the periodic part of the production is twice the grid-frequency, due to the quadratic dependence on the mean velocity gradient. In the continuity of the foregoing analysis, 
we will make several simplifications. As for the mean-field, we will assume the kinetic energy to reflect the periodicity of the production. We write, therefore,

$$
k(y)=k+A_{k} \cos (4 \pi y / M) .
$$

Furthermore we will assume the turbulent diffusivity again to be given by Eq. (12), and therefore independent of $y$, which is a very good approximation for $A_{k} / k \ll 1$. Finally, we will assume the dissipation to be uniform in the $y$ direction. These assumptions allow to evaluate the inhomogeneity of the kinetic energy as a balance between the inhomogeneous production and turbulent diffusion. The resulting expressions for the production and diffusion are then

$$
p(k)=p+\frac{K}{\tilde{M}^{2}} \frac{k^{2}}{\epsilon} \cos (4 \pi y / M)
$$

and

$$
d(y)=-\frac{2 A_{k}}{\tilde{M}^{2}} \frac{k^{2}}{\epsilon} \cos (4 \pi y / M) .
$$

Using these expressions, the equation for the amplitude of the variation of the kinetic energy is derived by substracting the equation for $k$ from the equation for $k(y)$, yielding

$$
\begin{aligned}
\dot{A_{k}} & =\frac{1}{\tilde{M}^{2}} \frac{k^{2}}{\epsilon}\left(K-2 A_{k}\right) \\
& =\alpha_{L}^{2} \frac{\epsilon}{k}\left(K-2 A_{k}\right) .
\end{aligned}
$$

The amplitude-equation, written in this simple form, reflects the balance between inhomogeneous production and cross-stream diffusion by turbulent fluctuations. It is clear from this balance that when the mean-field $K$ vanishes, the inhomogeneity will rapidly decay. Again it is the quantity $\alpha_{L}$, representing the length-scale ratio of turbulence scale to grid-scale, which pilots the dynamics.

\section{RESULTS}

\section{A. Choice of the parameters}

Equations (16)-(18) and (34) are integrated numerically in the high Reynolds number limit. The model constants are given their classical values [30] $C_{\epsilon 1}=1.44, C_{\epsilon 2}=1.92$, and $C_{v}=0.09$. The results we obtain depend obviously on the values of these coefficients, but the qualitative physics are fairly robust with respect to variations of these constants.

We have chosen initial conditions which are in the range of experimentally relevant values. To characterize the grid-spacing, we have chosen $M=10 \mathrm{~cm}$. The value of $U$ is typically an order of magnitude smaller than the mean flow velocity, which is of order $10 \mathrm{~ms}^{-1}$. We have therefore set the average-field kinetic energy to $K=1 \mathrm{~m}^{2} \mathrm{~s}^{-2}$. The initial value of the fluctuating quantities $k$ and $\epsilon$ are hard to estimate. Indeed, they depend on the precise instability mechanisms of the grid-bar wakes and the incoming turbulence intensity. We have therefore proceeded by taking two different values for $k$. We define the turbulence intensity by

$$
I_{k}=\frac{k}{K} .
$$

Our first set of computations corresponds to the intensity $I_{k}=0.1$. In the second set the initial velocity fluctuations are an order of magnitude smaller than the mean-field amplitude, $I_{k}=0.01$. We note here that this intensity corresponds to the intensity of the kinetic energy fluctuations upstream of the grid. The turbulence intensity measured in experiments corresponds to the maximum value of the kinetic energy measured downstream from the grid, and it is shown in the following how this downstream intensity depends on the initial conditions. 


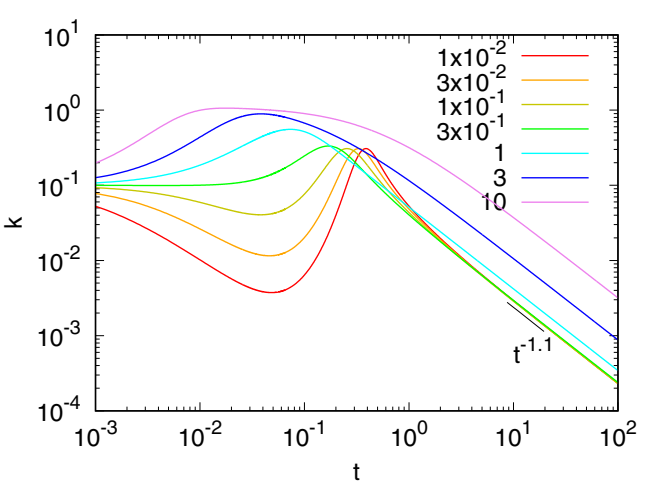

(a)

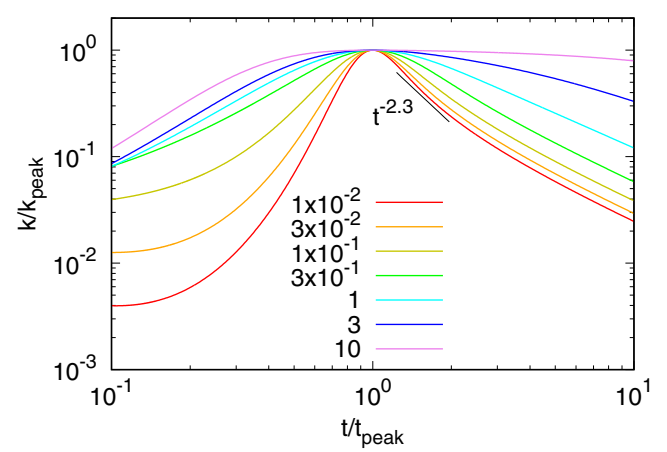

(c)

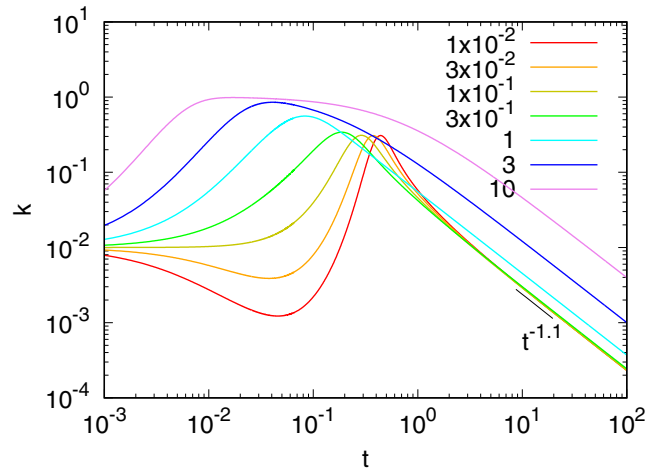

(b)

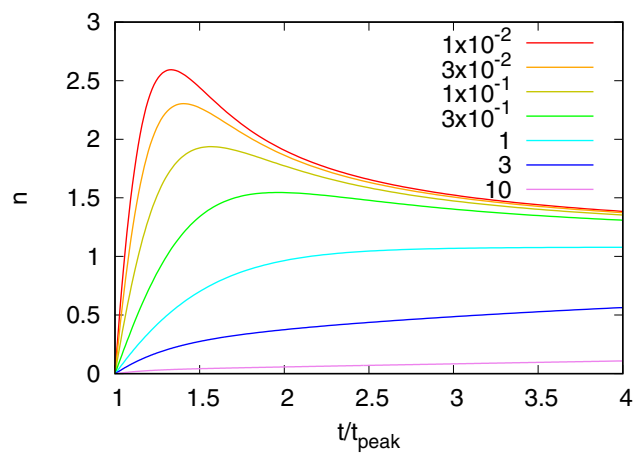

(d)

FIG. 2. Temporal evolution of the kinetic energy of the turbulence for values of the lenghtscale ratio $0.01 \leqslant$ $\alpha_{L}(0) \leqslant 10$. (a) Initial intensity $I_{k}=0.1$; (b) $I_{k}=0.01$. (c) Same results as in (b) but time is normalized by $t_{\text {peak }}$ and kinetic energy by $k_{\text {peak }}$. (d) Local power-law decay exponent of the kinetic energy $n$.

The initial values of $\epsilon$ are even harder to estimate than those of $k$. The variation of $\epsilon$ is in particular important, since for given $k$ and $M$, it determines the initial length-scale ratio $\alpha_{L}(0)$, defined in Eq. (22). The influence of this parameter on the dynamics constitutes one of the main issues of this investigation. Very large values of $\alpha_{L}(0)$ correspond to a strong transverse mixing of the turbulence near the grid as expected in active grids. Small initial values correspond to the case where the wakes are slowly perturbed by the developing turbulence, and the influence of the mean-flow, i.e., the wakes, is present far behind the grid. This corresponds to small initial turbulent intensity with a small initial lenght scale. Since the variation of this parameter is the key parameter in our study, we have varied it over three orders of magnitude, in the range $0.01 \leqslant \alpha_{L}(0) \leqslant 10$.

The initial values of $I_{k}$ and $\alpha_{L}$ are the physical control parameters of our system. The numerical integration of three coupled ODEs is performed using an explicit Euler integration scheme. The conservation of energy was estimated by monitoring the sum of the integral of the dissipation and the total kinetic energy in the system. Time steps were set to $\Delta t=10^{-4}$. A typical simulation of the system up to $t=100$ takes several seconds on a common desktop computer. More complicated numerics did not seem to be necessary.

\section{B. Evolution of the kinetic energy and dissipation}

In Figs. 2(a) and 2(b) we show the evolution of the turbulent kinetic energy for both turbulence intensities we studied, and a wide range of values of $\alpha_{L}(0)$. Clearly, it is shown that the results are not very sensitive with respect to the choice of the initial turbulent intensity, at least for the values 
$I_{k}=0.1$ and 0.01 which we considered. The influence of the initial length-scale ratio is observed to change importantly the short-time behavior, where the kinetic energy can either grow or decay, depending on the value of $\alpha_{L}(0)$. Also the peak value of the kinetic energy is affected by the initial conditions. The precise dependence of the peak-value and the peak-distance on the initial conditions will be discussed in Sec. VII. We further see that for long times the model behaves as expected, with a power-law decay

$$
k \sim t^{-n}
$$

where $n=1 /\left(C_{\epsilon 2}-1\right) \approx 1.1$.

In Fig. 2(c) the kinetic energy for $I_{k}=0.01$ is replotted, normalized by its peak-value. Time is normalized by $t_{\text {peak }}$, the time at which the kinetic energy peaks. Around the peak, the timedependence differs for the different cases. In particular, for small values of $\alpha_{L}(0)$ a power-law exponent steeper than the asymptotic value is observed. The local decay exponent, i.e., when the function is approximated by a power law, is

$$
n=-\frac{d \log (k)}{d \log (t)}
$$

This exponent is plotted in Fig. 2(d). It is observed that in the active grid limit [large $\alpha_{L}(0)$, the decay exponent monotonically increases from $n=0$ at the peak to $n \approx 1.1$ for long times. Only for small initial values of $\alpha_{L}$ an overshoot is observed with maximum values of the order of $n \approx 2.5$. Note that other exponents could be found by fitting power laws with a well chosen virtual origin. Such functional forms can fit the data over a longer range. A detailed discussion of different methods to choose such a virtual origin can be found in Refs. [12,35]. Nevertheless, at large distances downstream the grid the present model results will always converge to the asymptotic value $n=1 /\left(C_{\epsilon 2}-1\right)$, and this is also observed in experiments on different types of grid turbulence [20].

The mean-flow energy evolution is shown in Fig. 3(a). We observe, that irrespective of the parameters, the mean-energy decays rapidly, reducing its value by approximately 10 orders of magnitude in the interval $0<t<1$. It is thus in this interval that the influence of the production of kinetic energy by the shear-layer decreases from order unity to negligible. Clearly, it is this very fast decay of the mean field that makes grid-turbulence such a good candidate to study freely decaying isotropic turbulence. In the limit of large $\alpha_{L}(0)$, the kinetic energy of the mean field is thus rapidly absorbed by the turbulence. For this limit the flow approaches the freely decaying limit for which an analytical solution exists. For further discussion of this analytical solution we refer to Appendix C.

The straight lines in this semilogarithmic representation suggest an exponential decay. In the present model this is not exactly the case. Indeed, in the absence of $p$ the dissipation is proportional to $t^{-(n+1)}$. From Eq. (16) we have that far enough behind the grid (where $k$ and $\epsilon$ decay as power laws),

$$
\frac{\dot{K}}{K} \sim k^{2} / \epsilon \sim t^{1-n},
$$

and since $n$ is close to one, an almost pure exponential is observed at later times.

In Fig. 3(b) we show the evolution of the dissipation of turbulent kinetic energy. The qualitative behavior ressembles that of the kinetic energy in most aspects. The overall shapes of the curves are similar. But since we fixed the initial value of $k$ and varied the value of $\alpha_{L}(0)$, the initial values of $\epsilon$ are not the same for the different runs so that the peak values of the dissipation behave differently from those of the kinetic energy plotted in Fig. 2. For long times the expected power law, proportional to $t^{-(n+1)}$ is observed in the region where $p$ is small compared to $\epsilon$. 


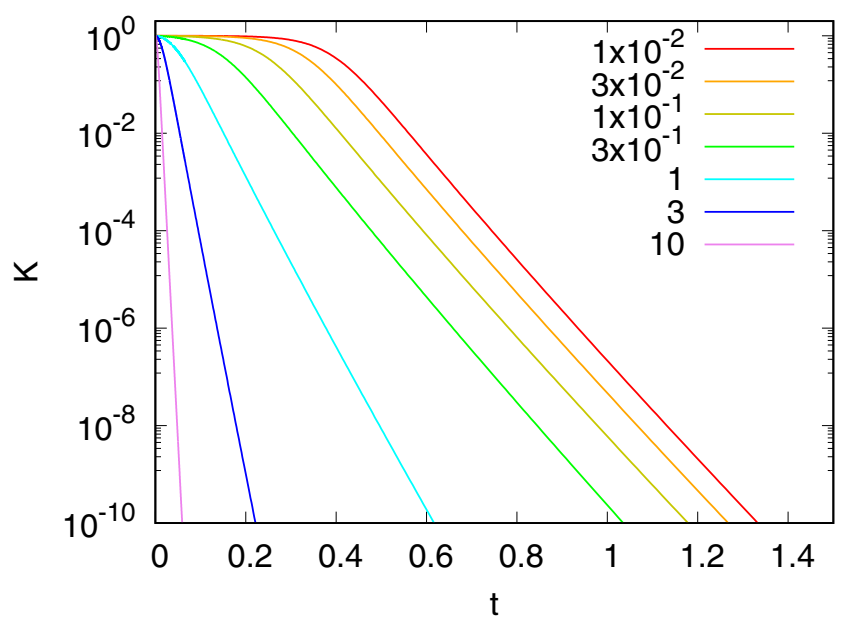

(a)

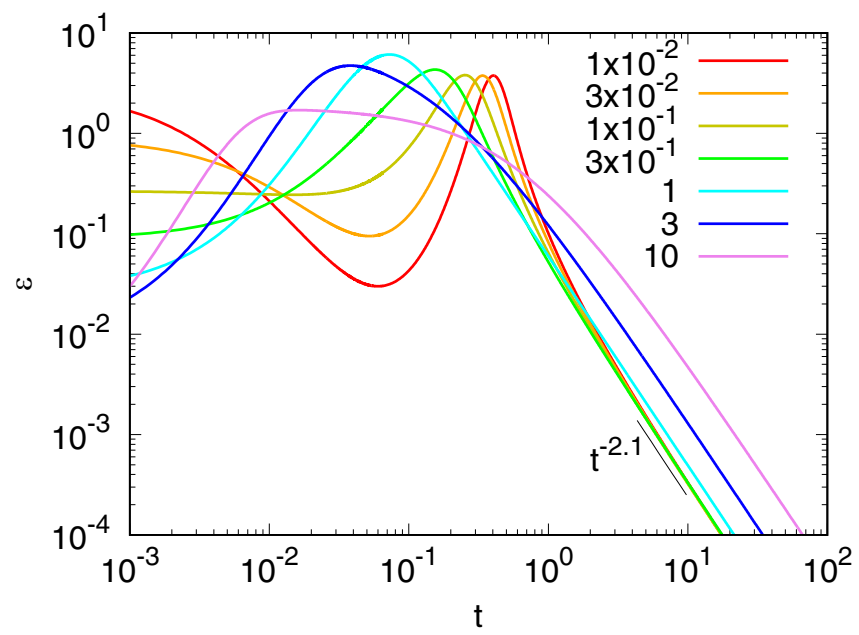

(b)

FIG. 3. Temporal evolution of (a) the kinetic energy of the mean field and (b) the dissipation of the turbulence, for values of the lenghtscale ratio $0.01 \leqslant \alpha_{L}(0) \leqslant 10$; initial intensity $I_{k}=0.01$.

\section{Nonequilibrium dissipation scaling}

The equilibrium value of $C_{\epsilon}$ [defined in Eq. (1)] is obtained when the production is equal to the dissipation. Such a steady state, which is in addition isotropic is hard to obtain experimentally, but in numerical simulations, forced isotropic turbulence in a periodic domain is a canonical flow [36]. The exact value might depend on the type of forcing, but for a given flow, for increasing $R_{\lambda}$ the value of $C_{\epsilon 0}$ tends to a constant value [37]. In freely decaying isotropic turbulence, the normalized dissipation rate at high Reynolds numbers tends to a different constant value, which depends weakly on the initial conditions [38]. Indeed in the case of decaying turbulence, the spectral shape at very small wave numbers determines the decay rate $n$, and modestly influences the exact value of $C_{\epsilon}$ [38].

The reason that in decaying turbulence a constant value of the normalized dissipation is observed, which is different from the value in forced turbulence, is that in the case of decaying turbulence, where $p$ goes to zero, the nonequilibrium will tend to an asymptotic value. In this self-similar state, 


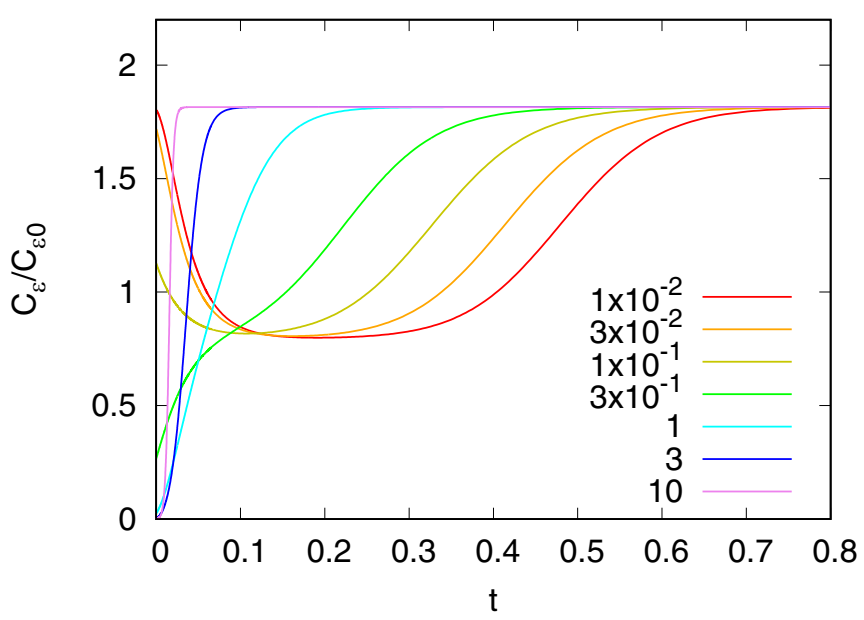

(a)

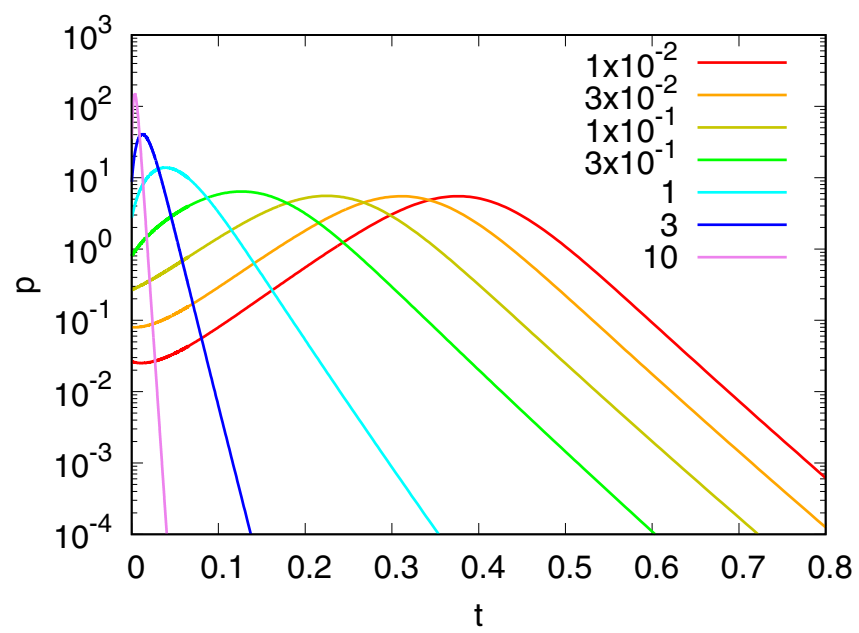

(b)

FIG. 4. (a) Normalized dissipation rate for values of the lenght-scale ratio $0.01 \leqslant \alpha_{L}(0) \leqslant 10$ and initial intensity $I_{k}=0.01$. (b) Production $p$ of turbulent kinetic energy.

the energy spectrum in the large scales will adapt to a constant shape, different from the one in the presence of a production mechanism. The shape of the modification of the energy spectrum in free decay can be obtained from perturbative analyis around the Kolmogorov spectrum [24,39,40]. Integrating this correction to determine the nonequilibrium value of the normalized dissipation rate yields [according to Eqs. (25) and (26)],

$$
\frac{C_{\epsilon}}{C_{\epsilon 0}}=\left(1-\frac{2}{9} C_{\epsilon 2}\right)^{-\frac{15}{14}} \approx 1.8 .
$$

In Fig. 4(a) we show our estimate of $C_{\epsilon} / C_{\epsilon 0}$, as determined by Eqs. (25) and (26). The transition from a monotonically rising value of $C_{\epsilon}$ to a plateau-value is abrubt. The physical reason for this is the rapid decrease of the production term as observed in Fig. 4(b). The production shows, as the mean-kinetic energy, a rapid decay at later times. The time at which this decay sets in is increasing with decreasing $\alpha_{L}(0)$. 


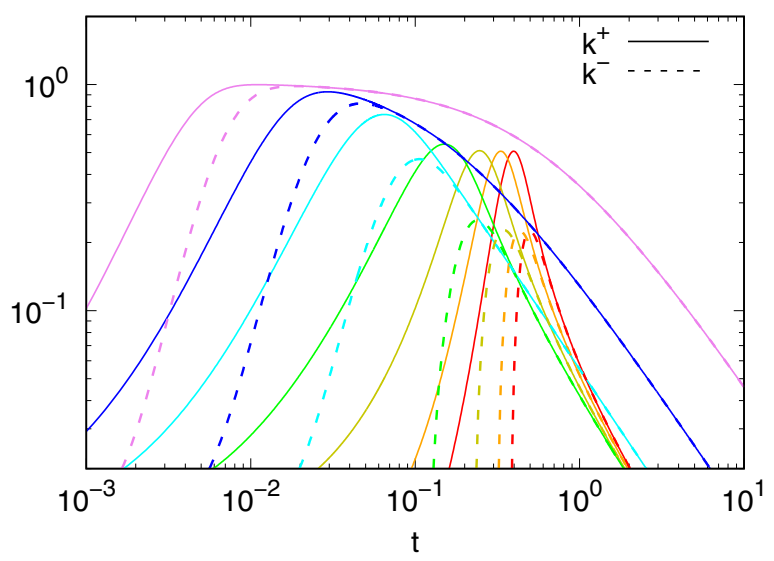

(a)

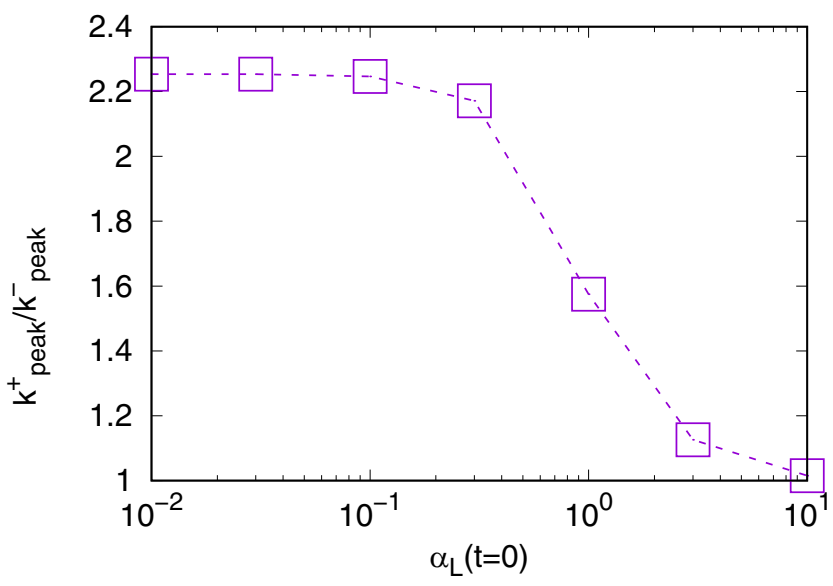

(b)

FIG. 5. Inhomogeneity of the kinetic energy induced by the periodic profile of the turbulence production term. (a) The maximum $\left(k^{+}=k+A_{k}\right.$; solid lines) and minimum values $\left(k^{-}=k-A_{k}\right.$; dashed lines) of the kinetic energy are shown for values of the lenghtscale ratio $0.01 \leqslant \alpha_{L}(0) \leqslant 10$ and initial intensity $I_{k}=0.01$. Line types as in the previous figures. (b) The peak of the kinetic energy $k^{+}$at the cross-stream $(y)$ position where the kinetic energy is maximum, divided by its peak at the $y$ position where the kinetic energy is minimum.

In the present flow, the constancy of the normalized dissipation, observed far enough behind the flow, corresponds thus to the vanishing of shearlayers and their associated production $p$. Beyond this downstream point the turbulence can therefore be considered statistically homogeneous.

\section{Cross-stream inhomogeneity}

The cross-stream-inhomogeneity, as modeled by Eq. (34) is shown in Fig. 5. In Fig. 5(a) we show how the kinetic energy evolves at two cross-stream positions behind the grid, one position where the production and thereby the kinetic energy is maximum $\left(k^{+}=k+A_{k}\right)$, the other one where the kinetic energy attains its minimum $\left(k^{-}=k-A_{k}\right)$. It is observed that in all cases, far behind the grid, the kinetic energy becomes statistically homogeneous so that $k^{+} \approx k^{-}$. The time where this happens depends on the initial value of $\alpha_{L}$. In the active grid-limit, the inhomogeneity vanishes almost immediately behind the grid (see for instance Ref. [41]). In the other limit, the 
inhomogeneity persists for a longer time and the peaks of $k^{+}$and $k^{-}$differ by a factor of order 2. This inhomogeneity is further quantified in Fig. 5(b), where it is shown how the ratio of the peak-values at the different streamwise positions depends on the initial conditions.

Clearly, in the present flow, the inhomogeneity, production, and mean-kinetic energy are all very closely related. These quantities thus all depend in the same way on the initial conditions, and they all vanish far away behind the grid, where the flow becomes statistically homogeneous and where the nonequilibrium $\tilde{k} / k$ tends to a constant value.

\section{E. Comparison with experimental results}

The main goal of the present investigation is to illustrate in a qualitative way the main features underlying the nonequilibrium observations in grid-turbulence. We have for that reason chosen the simplest possible model allowing for nonequilibrium scaling in a simplified representation of gridturbulence. In that way we can illustrate which are the needed ingredients to reproduce the physical phenomena. We are thus aiming for a qualitative agreement. However, since the principal motivation of the present work were some puzzling experimental observations, we think it is valuable to attempt a quantitative comparison with these results. Therefore, in this section we compare our simulations with some experimental and DNS data. We stress that our goal is not to assess the performance of the $k-\epsilon$ model or eddy-viscosity models in general.

A first observation is that far behind the grid a power law is observed in our computations. In experiments the value of the power law exponent is in general $n \approx 1.2[14,17,20]$. We observe a slightly smaller value [Figs. 2(a) and 2(b)], determined by the value of $C_{\epsilon 2}$. We could have changed its value to $C_{\epsilon 2}=11 / 6$ to have this agreement, but we have preferred to keep the original values of the constants in the model [30] which are fine-tuned to get an optimal agreement for a wide range of flow-geometries.

A detailed comparison of the global quantities $k$ and $\epsilon$ with values from literature is complicated since very few experimental investigations have measured them in grid-turbulence. However, in a recent numerical investigation [42] the turbulence generated behind a grid of cylinders was studied and these quantities were reported. In Figs. 6(a) and 6(b) we compare the evolution of $k$ and $\epsilon$ (normalized by their peak values) to the DNS results. It is observed that just beyond the peak and for about half of a decade in time, the DNS values for $k$ and $\epsilon$ closely follow our results for $\alpha_{L}(0)=3$. After this time interval the kinetic energy and dissipation change their behavior in the DNS study since the Reynolds number becomes very small. Indeed, the Reynolds number based on the meanvelocity and the cylinder diameter in this investigation was of order 1200, which is small compared to the experimental values which are typically of order $10^{4}$. Our model assumes a large value of the Reynolds number and is therefore not designed to capture this final regime of decay, corresponding to the change of regime in the DNS results. However, the fact that the kinetic energy and dissipation are simultaneously agreeing with the present model for the same set of initial conditions provides confidence to our approach.

In Fig. 6(c), we compare the kinetic energy normalized by its peak-value at a cross-stream location, where the kinetic energy is minimum, to measurements (same data as $k^{-}$in Fig. 5). Note that we do not compare exactly the same quantities. The data reported in the experiments [27] concern the root-mean-square value of the longitudinal velocity-fluctuations whereas we consider the kinetic energy. The normalization by peak-values $k_{\text {peak }}$ and $t_{\text {peak }}$ does correct somewhat for this difference, but a detailed comparison of the averaged quantities in the experiment with our model-approach would be valuable. The experimental measurements are taken at the centerline between two grid bars, which is a location where the production is minimum. We therefore compare with $k^{-}$, where the production is minimum as well. We do thus not take into account the anisotropy in our comparison, but we do consider the inhomogeneity.

It is observed that the data-points from the experiments fall in between the model-predictions for $0.3<\alpha_{L}(0)<1$. At long times all cases show the same power-law decay, as can be observed 


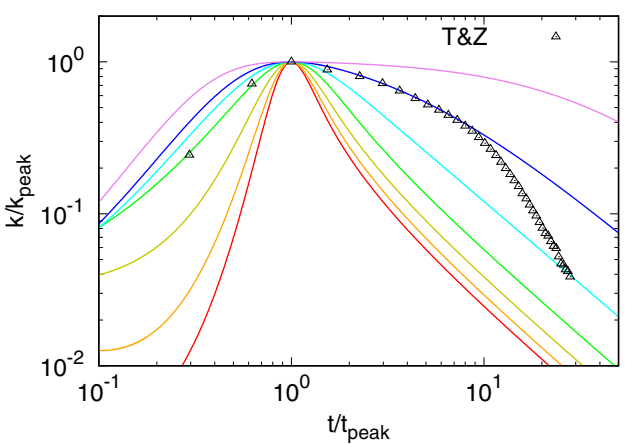

(a)

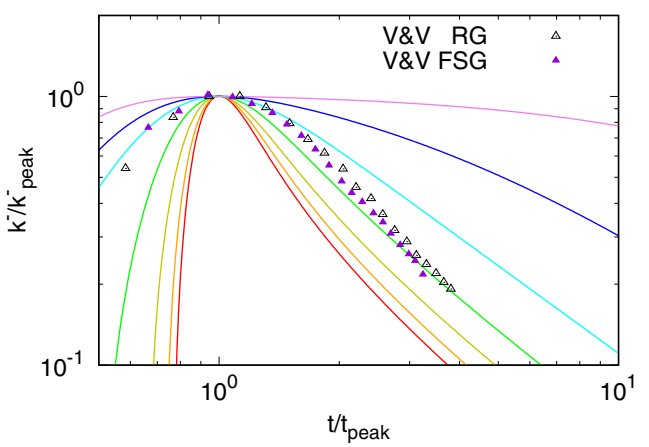

(c)

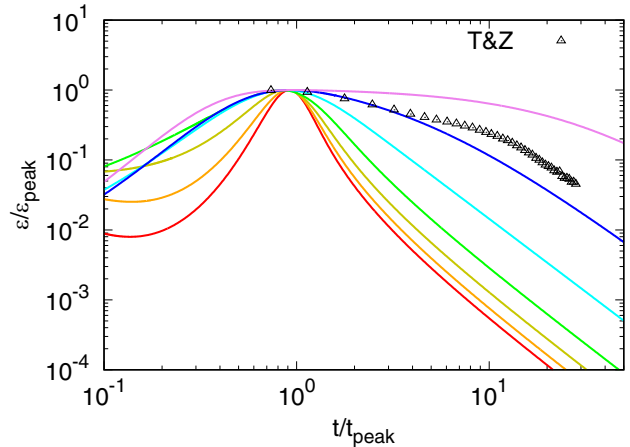

(b)

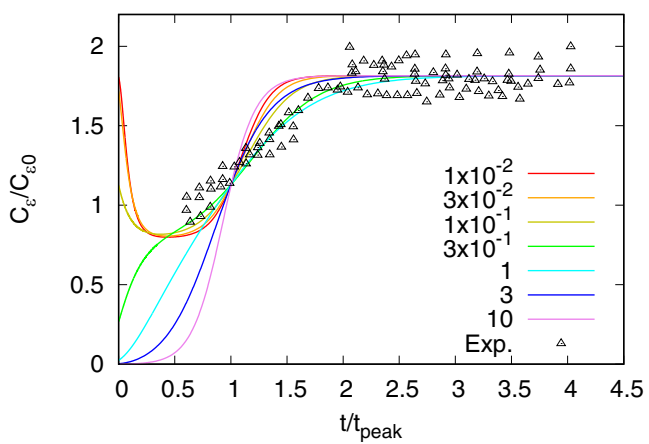

(d)

FIG. 6. (a) Temporal evolution of the kinetic energy of the turbulence for values of the length-scale ratio $0.01 \leqslant \alpha_{L}(0) \leqslant 10$ and $I_{k}=0.01$. (b) Evolution of the dissipation rate. Also shown in (a), (b) are DNS results of flow through an array of cylinders [42]. (c) Kinetic energy at a cross-stream position where the production of $k$ is minimum, compared with data obtained from a fractal square grid (FSG) and a regular square grid (RG) [27]. (d) Nonequilibrium of the flow as measured by the normalized dissipation, compared to experimental data-points [18].

in in Fig. 5(a). The steeper-than-expected decay of kinetic turbulence $(n>1.2)$ does not appear to persist for more than a decade, and is closely associated with the turbulence production and thereby the inhomogeneity of the flow. A similar conclusion was drawn in Ref. [43], who showed that the region where atypical decay behavior was observed (such as exponential time-dependence), corresponds to a region where the turbulence cannot be considered to be statistically homogeneous.

We have also compared to data of the normalized dissipation rate [18], Fig. 6(d), where different data-sets produced from wind-tunnel measurement behind a fractal grid are reported. Since we do not exactly know how to match the initial conditions of the experiments with our simulations, our comparison will be at best qualitative. Therefore, we have collapsed the asymtotic long-time value of 1.8 with the experimental data, and we have shifted the data-points horizontally (in time) to get a best agreement. This will only assess whether the transient toward the self-similar decaying state in our model shows the same time-dependence as the one observed in the experiments, but it will not assess the quantitative agreement. We observe that the best agreement is observed for the same set of initial conditions where best agreement was observed in Fig. 2(c), i.e., for values of $0.3<\alpha_{L}(0)<1$. This seems thus to be the correct order of magnitude for the initial length-scale ratio to model passive grid-turbulence.

The present results illustrate qualitatively how a simple model approach can capture the main features of turbulence in the nonequilibrium region behind a grid. A further investigation, focusing 


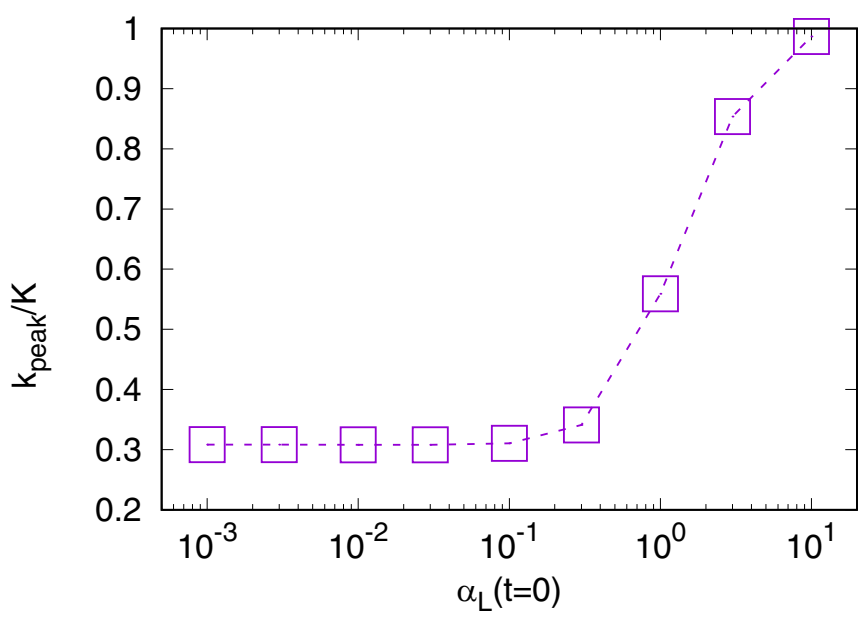

(a)

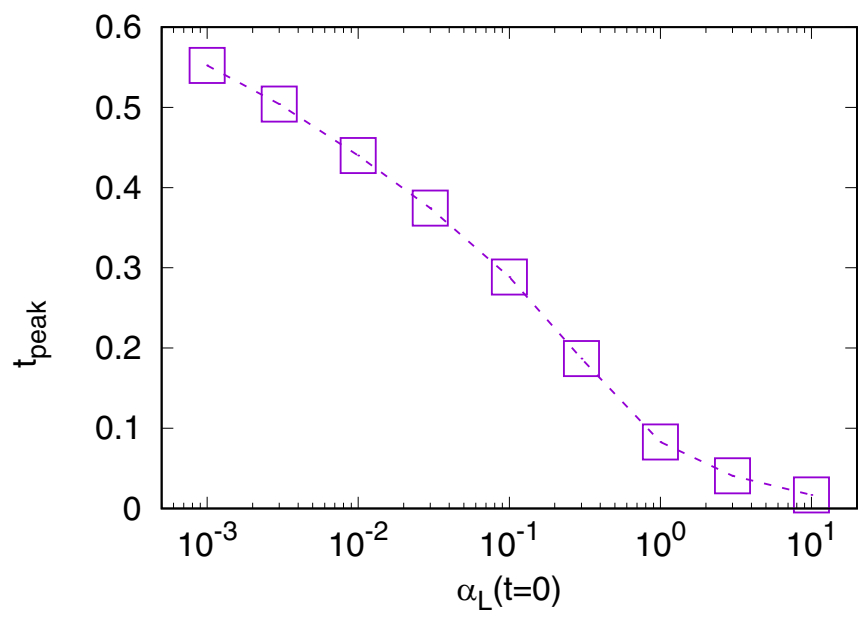

(b)

FIG. 7. (a) Peak values of the kinetic energy and (b) associated peak-times for $0.001 \leqslant \alpha_{L}(0) \leqslant 10$ and $I_{k}=0.01$.

on a pointwise comparison between DNS and model results, would be valuable to assess the flow in detail. Such a comparison is beyond the scope of the present investigation.

\section{SHORT-TIME EVOLUTION AND DETERMINATION OF THE PEAK-VALUE OF THE KINETIC ENERGY}

A remaining question is whether we can estimate how large a fraction of the kinetic energy of the mean field is converted into fluctuating kinetic energy.

In Fig. 7(a) we show how the peak of the kinetic energy of the turbulence depends on the parameter $\alpha_{L}(0)$. It is observed that for large values of $\alpha_{L}(0)$ almost all energy of the mean field $K$ is converted into turbulent fluctuations. This corresponds to the case where the length scale of the turbulence is initially large, so that the mean field is immediately distorted by the turbulent fluctuations. This limit corresponds in practice to active grid turbulence, where the rotating rods enhance drastically the cross-stream diffusion. 
When the initial fluctuations are initially very small, an asymptotic value of roughly $30 \%$ of the mean field energy is converted into turbulent fluctuations. The system seems to become independent on the size of the initial fluctuations for this limit. In Fig. 7(b) the location of the peak is determined. The quantity $t_{\text {peak }}$ corresponds to the time-instant where the kinetic energy attains its peak value. Obviously, this value cannot become negative. It is observed that in the limit where all the mean field energy is converted into fluctuations, the peak is located very close to the grid. For decreasing values of $\alpha_{L}(0)$ this distance is located further downstream. We will now explain these observations.

From Eq. (16) we can deduce the typical timescale over which the mean flow evolves,

$$
T=\left(\frac{1}{K} \frac{d K}{d t}\right)^{-1}=\frac{\epsilon \tilde{M}^{2}}{k^{2}} .
$$

The typical timescale of the turbulence is $\tau \equiv k / \epsilon$. Comparing these timescales we find that in the limit

$$
\frac{\tau}{T}=\frac{k^{3}}{\epsilon^{2} \tilde{M}^{2}} \ll 1,
$$

the mean flow evolves much slower than the turbulence. In this case the shear induced by the wakes can thus be treated as a steady shear. Note that this timescale ratio is the square of the length-scale ratio, $\alpha_{L}$.

In Fig. 8(a) we show how $\alpha_{L}$ evolves. Clearly for small initial values of $\alpha_{L}$, its value becomes of order unity approximately at $t=t_{\text {peak }}$, and before this time it is smaller. In the early times, the turbulent flow behaves thus as turbulence subjected to a steady shear. The average value of this shear is according to Eq. (15),

$$
S \sim \frac{K^{1 / 2}}{M}
$$

It is well known that the $k-\epsilon$ model applied to steady shear predicts exponential evolution of the kinetic energy and dissipation [31],

$$
\begin{aligned}
& k \sim k^{*} \exp (\mathrm{St}), \\
& \epsilon \sim \epsilon^{*} \exp (\mathrm{St}) .
\end{aligned}
$$

The values of $k^{*}$ and $\epsilon^{*}$ are set by the asymptotic value of $S^{*} \equiv S k^{*} / \epsilon^{*} \approx 3$. We can therefore estimate the time at which the shear-layers start to decay, by comparing

$$
\left.\frac{\tau}{T}\right|_{t=t_{*}}=\mathcal{O}(1)
$$

we find that

$$
t^{*} \sim \frac{1}{S} \ln \left(\frac{\tilde{M}^{2} \epsilon^{* 2}}{k^{* 3}}\right) .
$$

To find the peak value of the turbulent kinetic energy, we determine $k\left(t^{*}\right)$ by combining (46) and (43). This yields

$$
k\left(t^{*}\right) \sim \frac{\epsilon^{* 2} \tilde{M}^{2}}{k^{* 2}},
$$

which can be simplified to

$$
k\left(t^{*}\right) \sim \frac{K(t=0)}{S_{*}^{2}} .
$$




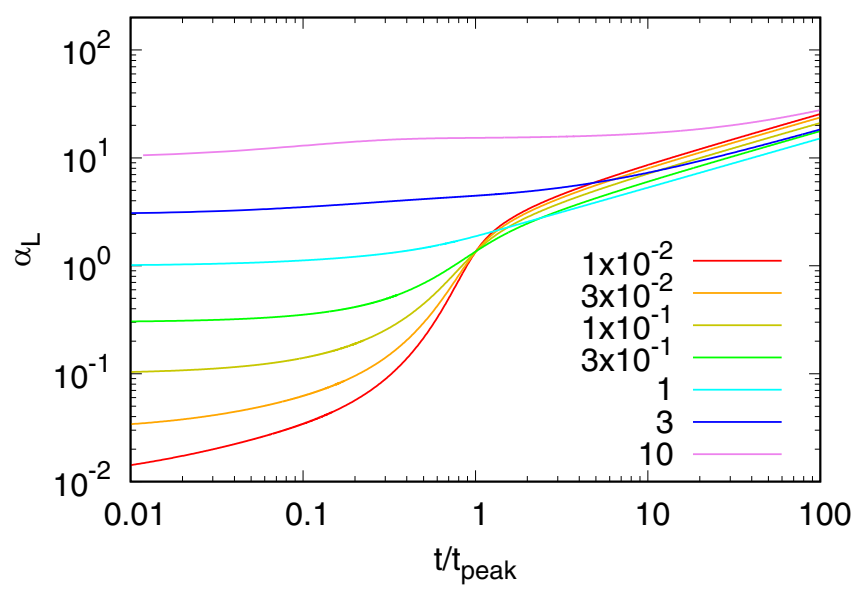

(a)

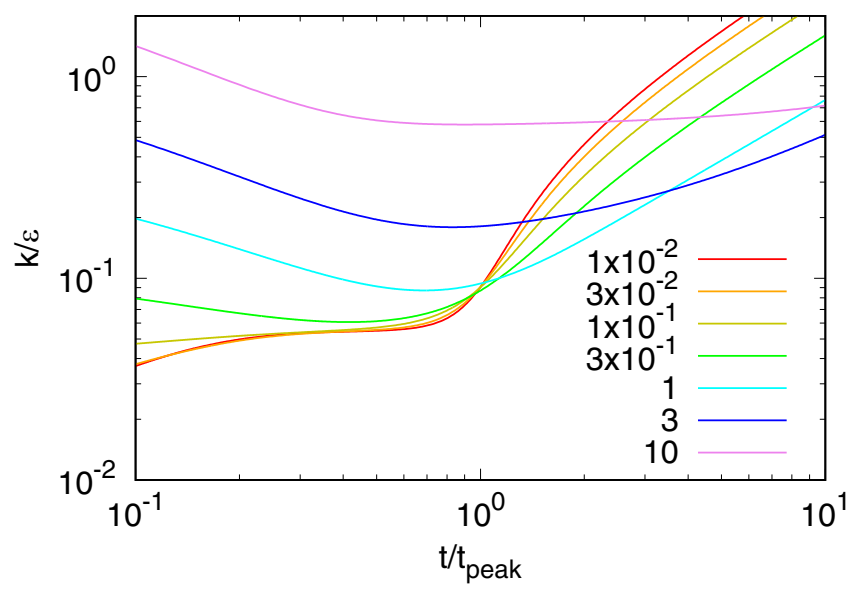

(b)

FIG. 8. Temporal evolution of (a) the lengthscale ratio and (b) the turbulent timescale $\tau=k / \epsilon$ for $0.01 \leqslant \alpha_{L}(0) \leqslant 10$ and $I_{k}=0.01$.

This shows that the peak of the turbulent kinetic energy corresponds to a fixed fraction of the total injected energy. In Fig. 8(b), we show the evolution of the turbulent timescale $\tau$. It is observed that for sufficiently small initial values of $\alpha_{L}(0), \tau$ is approximately constant for a short time-interval $0.2<t_{\text {peak }}<0.8$. It is in this region that exponential increase of the kinetic energy and dissipation [Eqs. (43) and (44)] is simultaneously observed.

The short-time evolution of the energy budget of the system can thus be accurately described as homogeneous, steady shearflow and the asymptotic behavior of this system sets the peak of the turbulent kinetic energy and the time instant where the peak occurs. This approximation is only valid for small initial values of $\alpha_{L}(0)$. We recall that the behavior in the other limit, of large initial values of $\alpha_{L}(0)$, is given by the analytical solution in Appendix C.

\section{NONEQUILIBRIUM IN PERIODIC BOX TURBULENCE}

As stated in the introduction, grid-turbulence was initially investigated as an experimental realization of almost isotropic turbulence. In numerical simulations, decaying isotropic turbulence 
is most easily investigated by considering the evolution of Navier-Stokes turbulence in a tripleperiodic box, developing from isotropic initial conditions.

It was observed in numerical simulations [44], that even in this context, in the absence of production, nonequilibrium scaling can be observed. The framework sketched in the foregoing, where the imbalance is associated with the difference between production and dissipation cannot be transposed directly to this case, since in the absence of production, the two-equation model used in this work will only reproduce the analytical solution derived in Appendix $\mathrm{C}$ and the dissipation rate constant $C_{\epsilon}$ will not change from its asymptotic value.

The main reason for this difference is that, to characterize the nonequilibrium, one needs two length scales (or timescales) corresponding to the energetic scales, which are in imbalance. For the grid-turbulence case, one of the scales is the scale of the shear-layers, the other one the integral scale of the turbulence. The reason that we can describe the present system so easily is that the shear-layers are fixed in at least one spatial direction, so that ensemble averaging allows to isolate them from the rest of the flow. In box-turbulence, when a large-scale contains a large amount of energy, this scale could be compared to some extent to a superposition of randomly oriented shear-layers. This scale, or coherent structure can then not isolated by spatial averaging, but it could be separated from the remainder of the flow by spectral filtering.

To describe the nonequilibrium features of purely isotropic turbulence without production, the model should thus be refined to take into account two scales. One way to do so is to consider a 2 -scale model. The simplest refined model which does this is the 2-scale model first proposed in Ref. [45], which writes in its simplest form,

$$
\begin{aligned}
\dot{k_{L}} & =p-f \\
\dot{k_{S}} & =f-\epsilon \\
\dot{f} & =\frac{f}{k_{L}}\left(C_{f 1} p-C_{f 2} f\right) \\
\dot{\epsilon} & =\frac{\epsilon}{k_{S}}\left(C_{\epsilon 1} f-C_{\epsilon 2} \epsilon\right)
\end{aligned}
$$

where the kinetic energy is divided into a large-scale part $k_{L}$ and a small scale part $k_{S}$. The sum of Eqs. (49) and (50) yields Eq. (8). Instead of measuring the imbalance by a difference between production and dissipation as in the foregoing, we now measure the difference between energy flux $f$ and energy dissipation $\epsilon$. Indeed the flux from the large scales to smaller scales can be compared to the energy flux from the shear-layers to the turbulence in the case of grid-turbulence.

In the absence of production, the case we are interested in here, three parameters need to be defined. We take the simplest form here where these parameters have constant values, suggested in the original paper: $C_{f 2}=1.8, C_{\epsilon 1}=1.08$ and $C_{\epsilon 2}=1.15$.

In Fig. 9 we show the results from an integration starting from initial conditions with energy concentrated in the large scales, $k_{L} / k_{S}=10^{2}$ and $f / \epsilon=10^{2}$. It is observed that at long times, the kinetic energy tends to its self-similar decay $k \sim t^{-n}$ and both the flux and the dissipation scale proportional to $t^{-(n+1)}$. However for times $t<10$, an important imbalance is observed where $\epsilon \neq f$. This imbalance can be represented by the change of the normalized dissipation rate. The perturbed energy can be computed, combining expressions (34) and (52), as

$$
\frac{\tilde{k}}{k}=\frac{2}{9} \frac{k}{k_{S}}\left(C_{\epsilon 1} \frac{f}{\epsilon}-C_{\epsilon 2}\right) .
$$

which yields using expression (26) the behavior in Fig. 9(b). We see that the refinement of the model allows to compute the imbalance in flows where the production is zero.

What is needed to take into account nonequilibrium is the existence of two separate length scales. In the presence of the mean-flow, as in grid-turbulence, the ratio of the two lenghtscales is given by 


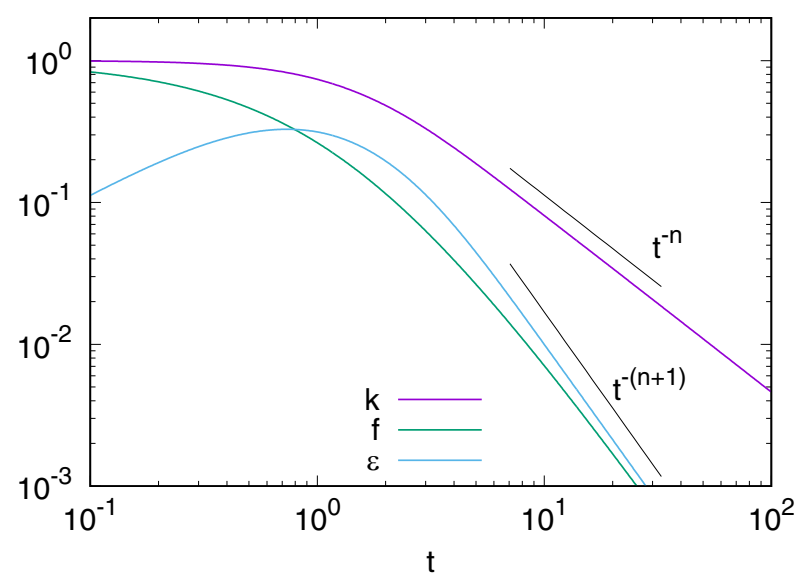

(a)

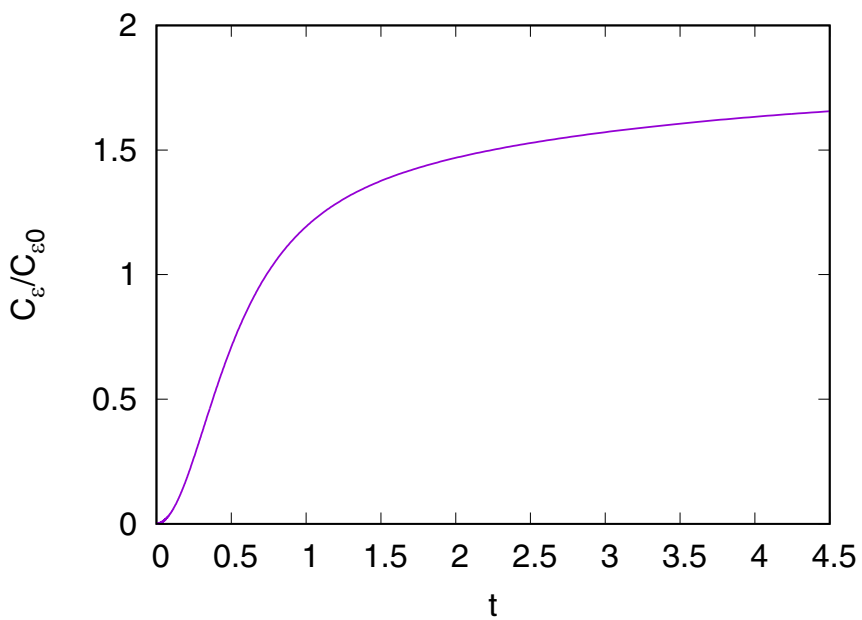

(b)

FIG. 9. Results from the two-scale model Eqs. (49)-(52). (a) Temporal evolution of the kinetic energy $k$, energy flux $f$ and dissipation rate $\epsilon$. (b) Evolution of the normalized dissipation rate.

$\alpha_{L}$. In the absence of a mean-flow it is the lenghtscale associated with the large-scale flux which can differ from the lenghtscale computed from the energy dissipation. To take into account this difference, at least a two-scale model is needed, which allows to measure the length-scale ratio $\epsilon / f$. In the absence of a two-scale description, the only parameters which determine the decay are the initial values of $k$ and $\epsilon$ and the decay will necessarily be given by the analytical solution described in Appendix C. In simulations and experiments, this extra length scale can possibly in certain cases be associated with coherent structures, an explanation proposed in [44] to account for the observed imbalance. Indeed, energy at a certain length scale can be represented by coherent structures, or shear-layers, the important point being that a certain scale contains an amount of energy larger (or smaller) than the value it would have in self-similar decay.

We have not tried to compare the results of the two-scale model more in detail with results from literature. Also, we do not claim that this is the most pertinent model. The goal of this article is not to show quantitative agreement with all possible nonequilibrium flows. What we do want to show is the general approach that allows to take into account nonequilibrium, and to measure and model it in an as simple manner as possible. Therefore we do not use the two-scale model in the foregoing 
discussion on grid-turbulence, since the shearlayers already allow to introduce a second length scale, and nonequilibrium can for that case already be measured using a standard two-equation model.

\section{CONCLUDING REMARKS}

The main goal of this investigation was to obtain a better understanding of the influence of initial conditions on grid turbulence and to show how the initial conditions influence the nonequilibrium properties of the downstream turbulence. To achieve this goal we have shown how to describe, statistically, the interaction of a mean field generated behind a grid with a spatially developing turbulent flow. The application of an eddy-viscosity model allowed to identify a parameter $\alpha_{L}$, proportional to the ratio of the mesh-size to the integral lenghtscale, which appears explicitly in the evolution equations of the energy budget. It is this $\alpha_{L}$ which is arguably large in active-grids, so that the influence of the initial conditions in turbulence generated by such grids is rapidly negligible. However for passive grids this parameter is smaller since the turbulence has to develop through the interaction with the shear layers. In such flows the influence of the initial conditions is expected to persist for longer time intervals.

The $k-\epsilon$ model turns out to be a convenient tool to understand and evaluate the energy budget in near-grid grid turbulence. That such a simple model can reproduce features of the experiment is due to several reasons. First of all, we consider integral quantities, such as the kinetic energy averaged over a period of the grid. This average allows to remove the influence of turbulent diffusion from our description. Secondly, the used model is known to give reasonable results for both shear flows and free decay. The present flow does not contain more complicated effects (mean swirl, rotation, pressure gradients, obstacles,...) and is therefore easier to predict than flows which do contain such features.

The present work does by no means prove that the $k-\epsilon$ model contains enough physics to describe more complicated flows. Already the choice of the model-parameters can be optimized [46], and more complicated flow features might need different models. For instance, strongly anisotropic flows would need at least the use of a Reynolds-stress closure [47]. Detailed insights into the energy cascade might need multi-scale descriptions such as the two-scale model used in the previous section, or a refinement of the model [48], or even more sophisticated closures such as the Direct Interaction Approximation [49] and its derivatives [50-52]. For instance, the use of such closures allows to reproduce the change of the far-stream decay exponent, by prescribing a particular shape of the energy spectrum at the large scales. The present model does not allow this freedom and the decay-exponent is determined by the value of $C_{\epsilon 2}$. Obviously, the choice of the simplest possible model cannot provide all the details of the flow. The choice of the model depends on the level of desired precision and the present work shows that several global-quantities in grid-turbulence do not need more complexity than the classical two-equation model to be qualitatively captured.

The insights obtained from the numerical integration of the model allow now to give answers to the questions which were raised in the introduction.

(1) The sudden transition from the power-law evolution of $C_{\epsilon}$ to a constant value is caused by the rapid decay of the production term in this zone. The turbulence evolves here from a state where the production is equal or larger than the dissipation, to a state where the production becomes negliglible.

(2) The downstream distance where this happens depends on the initial length-scale ratio $\alpha_{L}$. In particular, when this ratio is decreasing, the distance behind the grid where the kinetic energy peaks increases.

(3) For active grids this distance is arguably much smaller than for passive grids.

(4) The peak of the kinetic energy is naturally bounded by the total kinetic energy in the flow. The minimum fraction of the mean energy converted into turbulent kinetic energy tends to a constant of order 0.3 for small values of $\alpha_{L}(0)$.

We showed that in this latter limit, of small initial value of the length-scale ratio, the timedependence of the kinetic energy directly behind the peak of the kinetic energy is steeper than the 
asymptotic power-law exponent, the opposite trend being observed for large values of $\alpha_{L}(0)$. This same quantity pilots also the inhomogeneity of the production, and thereby the inhomogeneity of the cross-stream kinetic energy profile.

We showed that the nonequilibrium, as quantified by the normalized dissipation, can be evaluated a posteriori from the results of the $k-\epsilon$ model. The next challenge is to use this estimate to improve the performance of the model in cases where the nonequilibrium deteriorates its performance. One application is the modeling of turbulent flow around obstacles, since the nonequilibrium affects the spreading of the radial extent of the wake (see for example Ref. [53]). This seems an excellent subject for further research.

\section{APPENDIX A: DERIVATION OF THE EQUATIONS FOR $K$ AND $k$}

We start from the incompressible Navier-Stokes equations,

$$
\partial_{t} U_{i}+U_{j} \partial_{j} U_{i}=-\partial_{i} \Pi+v \partial_{j}^{2} U_{i}
$$

and $\partial_{i} U_{i}=0$. We decompose the flow into an ensemble averaged part and a fluctuating part $U_{i}=\bar{U}_{i}+u_{i}$ and the pressure divided by density is $\Pi=\bar{\Pi}+\pi$. The three components of the fluctuating velocity are indicated by $\left(u_{1}, u_{2}, u_{3}\right)=(u, v, w)$. Furthermore we consider the case where the mean flow is unidirectional, homogeneous in the $\mathrm{x}$-direction and varying in only one direction $y$ perpendicular to the flow direction $x$,

$$
\bar{U}_{i}=\bar{U}(y) \delta_{i 1} .
$$

For grid-turbulence this means that we are moving in a reference frame with constant velocity $U_{0}$. The equation for the mean field then reads

$$
\partial_{t} \bar{U}=-\partial_{j} \overline{u u_{j}}+v \partial_{y}^{2} \bar{U} .
$$

The equation for the fluctuations reads,

$$
\partial_{t} u_{i}+\bar{U} \partial_{x} u_{i}+v \partial_{y} \bar{U} \delta_{i 1}=-\partial_{j}\left(\delta_{i j} \pi+u_{i} u_{j}\right)+v \partial_{j}^{2} u_{i}
$$

so that the kinetic energy of the mean field and of the fluctuations obey

$$
\frac{1}{2} \partial_{t} \bar{U}^{2}=-\partial_{j}\left(\bar{U} \overline{u u_{j}}\right)+\overline{u v} \partial_{y} \bar{U}+v \partial_{y}^{2}\left(\frac{1}{2} \bar{U}^{2}\right)-v\left(\partial_{y} \bar{U}\right)^{2},
$$

and

$$
\frac{1}{2} \partial_{t} \overline{u_{i} u_{i}}+\frac{1}{2} \bar{U} \partial_{x} \overline{u_{i} u_{i}}+\overline{u v} \partial_{y} \bar{U}=-\partial_{j}\left(\delta_{i j} \overline{u_{i} \pi}+\frac{1}{2} \overline{u_{i} u_{i} u_{j}}\right)+v \partial_{j}^{2}\left(\frac{1}{2} \overline{u_{i} u_{i}}\right)-v \overline{\partial_{j} u_{i} \partial_{j} u_{i}} .
$$

The turbulence is considered statistically homogeneous in the $x-$ and $z$-direction. All gradients of averaged quantities then vanish in these directions, leading to

$$
\frac{1}{2} \partial_{t} \bar{U}^{2}=-\partial_{y}(\bar{U} \overline{u v})+\overline{u v} \partial_{y} \bar{U}+v \partial_{y}^{2}\left(\frac{1}{2} \bar{U}^{2}\right)-v\left(\partial_{y} \bar{U}\right)^{2},
$$

and

$$
\frac{1}{2} \partial_{t} \overline{u_{i} u_{i}}=\underbrace{-\overline{u v} \partial_{y} \bar{U}}_{p(y)}-\underbrace{-\partial_{y}\left(\overline{v \pi}+\frac{1}{2} \overline{u_{i} u_{i} v}\right)+v \partial_{y}^{2}\left(\frac{1}{2} \overline{u_{i} u_{i}}\right)}_{d(y)}-\underbrace{v \overline{\partial_{j} u_{i} \partial_{j} u_{i}}}_{\epsilon(y)} .
$$

On the right hand side of this last equation we have the three different contributions to the kinetic energy, production $p(y)$, diffusion $d(y)$ and dissipation $\epsilon(y)$. These two equations give the precise, pointwise evolution of the kinetic energy of the flow with a cross-stream mean profile, in a reference frame moving with the mean-velocity. If we want to simplify, we have to know more on the meanprofile. In a periodic grid, of whatever type with a periodic spacing, the mean field will be periodic. 
Integrating the kinetic energy over one period, all terms which can be written as the gradient in the $y$ direction will cancel out. Defining the average kinetic energies,

$$
\begin{aligned}
& K=\frac{1}{2 M} \int_{0}^{M} \bar{U}^{2} d y, \\
& k=\frac{1}{2 M} \int_{0}^{M} \overline{u_{i} u_{i}} d y,
\end{aligned}
$$

we have

$$
\dot{K}=-p-D
$$

and

$$
\dot{k}=p-\epsilon
$$

with

$$
\begin{aligned}
p & =-\frac{1}{M} \int_{0}^{M} \overline{u v} \partial_{y} \bar{U} d y, \\
\epsilon & =\frac{v}{M} \int_{0}^{M} \overline{\partial_{j} u_{i} \partial_{j} u_{i}} d y, \\
D & =\frac{v}{M} \int_{0}^{M}\left(\partial_{y} \bar{U}\right)^{2} d y .
\end{aligned}
$$

Equations (A11) and (A12) are thus exact for flows with a mean flow which is unidirectional and homogeneous in the streamwise direction and with a transverse periodic variation.

\section{APPENDIX B: ASSESSMENT OF THE INFLUENCE OF INHOMOGENEITY OF $k$ AND $\epsilon$ ON THE MODEL PREDICTIONS}

In this section we will assess the error induced by the assumption that the turbulent kinetic energy and its dissipation are homogeneously distributed in the transverse $(y)$ direction.

We indicate for brevity the transverse average of a quantity

$$
\langle F\rangle \equiv \frac{1}{M} \int_{0}^{M} F d y .
$$

In the model equations, the production term was determined by

$$
p=C_{\nu} \frac{k^{2}}{\epsilon}\left\langle\left(\partial_{y} \bar{U}\right)^{2}\right\rangle
$$

Strictly speaking, the transverse average should also concern the quantities $k, \epsilon$, but this would complicate the resolution of the ODE model and we have therefore neglected this transverse dependence of $k$ and $\epsilon$.

We check here the error induced by this assumption, by computing the ratio,

$$
R_{p}=\frac{\left\langle\frac{k_{y}^{2}}{\epsilon_{y}} S(y)^{2}\right\rangle}{\frac{k^{2}}{\epsilon}\left\langle S(y)^{2}\right\rangle},
$$

where $k_{y}, \epsilon_{y}$ are the pointwise, $y$-dependent kinetic energy and dissipation, respectively. By definitions $k=\left\langle k_{y}\right\rangle$ and $k=\left\langle\epsilon_{y}\right\rangle$. The validity of the approximation of homogeneous $k$ and $\epsilon$ depends then on the $y$-dependence of $k_{y}$ and $\epsilon_{y}$.

We assume, as for the mean velocity a periodic distribution for $k_{y}$ and $\epsilon_{y}$,

$$
k_{y}=k\left[1+\gamma_{k} \cos \left(\frac{4 \pi y}{M}\right)\right], \quad \epsilon_{y}=\epsilon\left[1+\gamma_{\epsilon} \cos \left(\frac{4 \pi y}{M}\right)\right],
$$




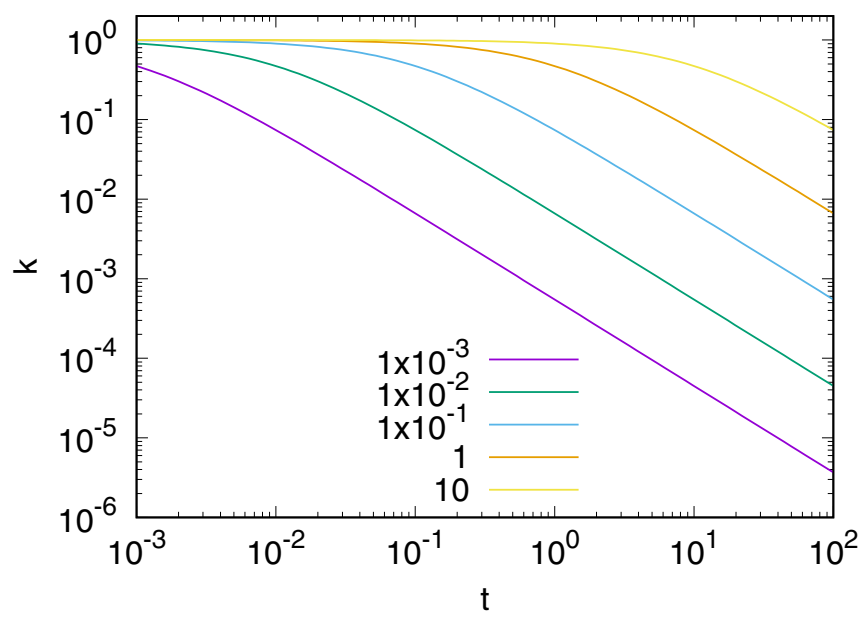

FIG. 10. Analytical solution for the temporal evolution of the turbulent kinetic energy for freely decaying turbulence for different initial values of the ratio $t_{0}=k(0) / \epsilon(0)$.

where the spatial frequency is chosen to match the frequency of the squared mean-velocity gradient. From the experimental results [27], Figs. 6(a) and 6(d), we observe that the values of $\gamma_{k}$ and $\gamma_{\epsilon}$ do not exceed 0.3 for $x>1.8 x_{\text {peak }}$ and that in most of the nonequilibrium region this value is significantly lower, $\gamma<0.1$. Using these maximum values, the absolute value of $R_{p}$ does never exceed 3\%. Two other quantities appear in the $\epsilon$ equation, $k S^{2}$ and $\epsilon^{2} / k$. For these two terms we have also verified the error induced by the assumption of homogeneity and the errors induced are similarly never exceeding 3\%. The assumption that we can use the values of $k$ and $\epsilon$ instead of $k_{y}$ and $\epsilon_{y}$ in the model are valid within a few percent.

\section{APPENDIX C: ANALYTICAL SOLUTION FOR THE KINETIC ENERGY IN THE ACTIVE GRID LIMIT}

The total energy of the flow, corresponding to both the fluctuating energy plus the mean field does not have a source term once the flow has passed the grid. Indeed, summing Eqs. (A11) and (A12), the overall budget is evolving according to

$$
\dot{K}_{\text {tot }}=-\epsilon_{\text {tot }},
$$

where $K_{\text {tot }}=K+k$ and $\epsilon_{\text {tot }}=D+\epsilon$. In principle, in the present investigation $D$ can be neglected compared to $\epsilon$. The $k-\epsilon$ model reduces now to

$$
\begin{aligned}
\dot{K}_{\text {tot }} & =-\epsilon, \\
\dot{\epsilon} & =-C_{\epsilon 2} \frac{\epsilon^{2}}{k} .
\end{aligned}
$$

This system is not closed, and in the present investigation we have modeled the repartition of energy between $K$ and $k$. Let us now consider the limiting case, which was identified for $\alpha_{L}(0) \gg 1$. In this case, which we coined the active grid limit, the energy is almost immediately transferred to the turbulence such that $K_{\text {tot }} \approx k$. For this case the above system has an analytical solution

$$
\frac{k}{k(0)}=\left(\frac{t+t_{0}}{t_{0}}\right)^{-n},
$$

with $n=1 /\left(C_{\epsilon 2}-1\right)$ and $t_{0}=n k(0) /[\epsilon(0)]$. This expression is plotted in Fig. 10, for different values of $t_{0}$. 
[1] L. F. G. Simmons and C. Salter, Experimental investigation and analysis of the velocity variations in turbulent flow, Proc. R. Soc. London Ser. A 145, 212 (1934).

[2] G. I. Taylor, Statistical theory of turbulence, Proc. R. Soc. London Ser. A 151, 421 (1935).

[3] R. B. Cal, J. Lebrón, L. Castillo, H. S. Kang, and C. Meneveau, Experimental study of the horizontally averaged flow structure in a model wind-turbine array boundary layer, J. Renew. Sustain. Energy 2, 013106 (2010).

[4] S. Rockel, J. Peinke, M. Hölling, and R. B. Cal, Wake to wake interaction of floating wind turbine models in free pitch motion: An eddy viscosity and mixing length approach, Renew. Energy 85, 666 (2016).

[5] K. Yoon and Z. Warhaft, The evolution of grid-generated turbulence under conditions of stable thermal stratification, J. Fluid Mech. 215, 601 (1990).

[6] L. Danaila, F. Anselmet, T. Zhou, and R. Antonia, A generalization of Yaglom's equation which accounts for the large-scale forcing in heated decaying turbulence, J. Fluid Mech. 391, 359 (1999).

[7] L. Mydlarski and Z. Warhaft, Passive scalar statistics in high Peclet number grid turbulence, J. Fluid Mech. 358, 135 (1998).

[8] S. Ayyalasomayajula, A. Gylfason, L. R. Collins, E. Bodenschatz, and Z. Warhaft, Lagrangian Measurements of Inertial Particle Accelerations in Grid Generated Wind Tunnel Turbulence, Phys. Rev. Lett. 97, 144507 (2006).

[9] M. Obligado, T. Teitelbaum, A. Cartellier, P. Mininni, and M. Bourgoin, Preferential concentration of heavy particles in turbul., J. Turbulence 15, 293 (2014).

[10] S. R. Stalp, L. Skrbek, and R. J. Donnelly, Decay of Grid Turbulence in a Finite Channel, Phys. Rev. Lett. 82, 4831 (1999).

[11] G. Comte-Bellot and S. Corrsin, The use of contraction to improve the isotropy of grid-generated turbulence, J. Fluid Mech. 25, 657 (1966).

[12] M. Mohamed and J. Larue, The decay power law in grid-generated turbulence, J. Fluid Mech. 219, 195 (1990).

[13] H. E. Cekli and W. van de Water, Tailoring turbulence with an active grid, Exp. Fluids 49, 409 (2010).

[14] M. Sinhuber, E. Bodenschatz, and G. P. Bewley, Decay of Turbulence at High Reynolds Numbers, Phys. Rev. Lett. 114, 034501 (2015).

[15] A. Fuchs, S. M. Duarte Queirós, P. G. Lind, A. Girard, F. Bouchet, M. Wächter, and J. Peinke, Small-scale structures of turbulence in terms of entropy and fluctuation theorems, Phys. Rev. Fluids 5, 034602 (2020).

[16] G. K. Batchelor and A. A. Townsend, Decay of isotropic turbulence in the initial period, Proc. R. Soc. London A 193, 539 (1948).

[17] L. Mydlarski and Z. Warhaft, On the onset of high-Reynolds-number grid-generated wind tunnel turbulence, J. Fluid Mech. 320, 331 (1996).

[18] J. C. Vassilicos, Dissipation in turbulent flows, Annu. Rev. Fluid Mech. 47, 95 (2015).

[19] H. Tennekes and J. Lumley, A First Course in Turbulence (MIT Press, Cambridge, MA, 1972).

[20] P.-A. Krogstad and P. Davidson, Freely decaying, homogeneous turbulence generated by multi-scale grids, J. Fluid Mech. 680, 417 (2011).

[21] K. Nagata, Y. Sakai, T. Inaba, H. Suzuki, O. Terashima, and H. Suzuki, Turbulence structure and turbulence kinetic energy transport in multiscale/fractal-generated turbulence, Phys. Fluids 25, 065102 (2013).

[22] S. Discetti, I. B. Ziskin, T. Astarita, R. Adrian, and K. P. Prestridge, Piv measurements of anisotropy and inhomogeneity in decaying fractal generated turbulence, Fluid Dyn. Res. 45, 061401 (2013).

[23] A. Thormann and C. Meneveau, Decay of homogeneous, nearly isotropic turbulence behind active fractal grids, Phys. Fluids 26, 025112 (2014).

[24] W. J. T. Bos and R. Rubinstein, Dissipation in unsteady turbulence, Phys. Rev. Fluids 2, 022601(R) (2017).

[25] M. Meldi and P. Sagaut, Investigation of anomalous very fast decay regimes in homogeneous isotropic turbulence, J. Turbulence 19, 390 (2018).

[26] O. Reynolds, IV. On the dynamical theory of incompressible viscous fluids and the determination of the criterion, Philos. Trans. R. Soc. London A 186, 123 (1895).

[27] P. Valente and J. C. Vassilicos, The nonequilibrium region of grid-generated decaying turbulence, J. Fluid Mech. 744, 5 (2014). 
[28] H. Makita, Realization of a large-scale turbulence field in a small wind tunnel, Fluid Dyn. Res. 8, 53 (1991).

[29] L. Mydlarski, A turbulent quarter century of active grids: From Makita (1991) to the present, Fluid Dyn. Res. 49, 061401 (2017).

[30] W. Jones and B. E. Launder, The prediction of laminarization with a two-equation model of turbulence, Int. J. Heat Mass Transf. 15, 301 (1972).

[31] S. Pope, in Turbulent Flows (Cambridge University Press, Cambridge, UK, 2000), p. 350.

[32] R. Rubinstein, Formulation of a Two-scale Model of Turbulence, Tech. Rep. (ICASE, Hampton, VA, 2000).

[33] W. K. George, The decay of homogeneous isotropic turbulence, Phys. Fluids A 4, 1492 (1992).

[34] P. Spalart and S. Allmaras, A one-equation turbulence model for aerodynamic flows, in Proceedings of the 30th Aerospace Sciences Meeting and Exhibit (1992), p. 439.

[35] P. Valente and J. C. Vassilicos, The decay of turbulence generated by a class of multiscale grids, J. Fluid Mech. 687, 300 (2011).

[36] T. Ishihara, T. Gotoh, and Y. Kaneda, Study of high Reynolds number isotropic turbulence by Direct Numerical Simulation, Annu. Rev. Fluid Mech. 41, 165 (2009).

[37] Y. Kaneda, T. Ishihara, M. Yokokawa, K. Itakura, and A. Uno, Energy dissipation rate and energy spectrum in high resolution direct numerical simulations of turbulence in a periodic box, Phys. Fluids 15, L21 (2003).

[38] W. J. T. Bos, L. Shao, and J.-P. Bertoglio, Spectral imbalance and the normalized dissipation rate of turbulence, Phys. Fluids 19, 045101 (2007).

[39] A. Yoshizawa, Nonequilibrium effect of the turbulent-energy-production process on the inertial-range energy spectrum, Phys. Rev. E 49, 4065 (1994).

[40] K. Horiuti and T. Tamaki, Nonequilibrium energy spectrum in the subgrid-scale one-equation model in large-eddy simulation, Phys. Fluids 25, 125104 (2013).

[41] S. Weitemeyer, N. Reinke, J. Peinke, and M. Hölling, Multi-scale generation of turbulence with fractal grids and an active grid, Fluid Dyn. Res. 45, 061407 (2013).

[42] S. Tao and Y. Zhou, Turbulent flows around side-by-side cylinders with regular and multiscale arrangements, Phys. Rev. Fluids 4, 124602 (2019).

[43] P.-Å. Krogstad and P. Davidson, Near-field investigation of turbulence produced by multi-scale grids, Phys. Fluids 24, 035103 (2012).

[44] S. Goto and J. C. Vassilicos, Unsteady turbulence cascades, Phys. Rev. E 94, 053108 (2016).

[45] K. Hanjalic, B. Launder, and R. Schiestel, Multiple-timescale concepts in turbulent transport modeling, in Von Karman Institute for Fluid Dynamics Measurements and Predictions of Complex Turbulent Flows, Vol. 1 (1980), p. 35.

[46] L. Margheri, M. Meldi, M. Salvetti, and P. Sagaut, Epistemic uncertainties in RANS model free coefficients, Comput. Fluids 102, 315 (2014).

[47] B. E. Launder, G. Reece, and W. Rodi, Progress in the development of a Reynolds-stress turbulence closure, J. Fluid Mech. 68, 537 (1975).

[48] A. Cadiou, K. Hanjalić, and K. Stawiarski, A two-scale second-moment turbulence closure based on weighted spectrum integration, Theor. Comput. Fluid Dyn. 18, 1 (2004).

[49] R. H. Kraichnan, The structure of isotropic turbulence at very high Reynolds numbers, J. Fluid Mech. 5, 497 (1959).

[50] S. A. Orszag, Analytical theories of turbulence, J. Fluid Mech. 41, 363 (1970).

[51] R. H. Kraichnan, An almost-Markovian Galilean-invariant turbulence model, J. Fluid Mech. 47, 513 (1971).

[52] W. J. T. Bos and J.-P. Bertoglio, Lagrangian Markovianized field approximation for turbulence, J. Turbul. 14, 99 (2013).

[53] M. Obligado, T. Dairay, and J. C. Vassilicos, Nonequilibrium scalings of turbulent wakes, Phys. Rev. Fluids 1, 044409 (2016). 